\title{
A SYSTEM OF INTERACTION AND STRUCTURE II: THE NEED FOR DEEP INFERENCE
}

\author{
ALWEN TIU \\ Computer Science Laboratory, Research School in Information Science and Engineering, Australian \\ National University, Canberra ACT 0200, Australia. \\ e-mail address: Alwen.Tiu@rsise.anu.edu.au
}

\begin{abstract}
This paper studies properties of the logic BV, which is an extension of multiplicative linear logic (MLL) with a self-dual non-commutative operator. BV is presented in the calculus of structures, a proof theoretic formalism that supports deep inference, in which inference rules can be applied anywhere inside logical expressions. The use of deep inference results in a simple logical system for MLL extended with the self-dual noncommutative operator, which has been to date not known to be expressible in sequent calculus. In this paper, deep inference is shown to be crucial for the logic BV, that is, any restriction on the "depth" of the inference rules of BV would result in a strictly less expressive logical system.
\end{abstract}

\section{INTRODUCTION}

This paper is the second part of a planned series of papers on the calculus of structures, started in Gug02. The calculus of structures is a new formalism for presenting logics. It is a generalization of the one-sided sequent calculus (also known as Gentzen-Shütte sequent calculus [TS96]). One of the main features of the calculus of structures is that it allows deep inference, i.e., inference rules in the calculus of structures can be applied arbitrarily deep inside logical expressions. This is in contrast with the traditional logical rules in sequent calculus, as in, e.g., Gentzen's LK [Gen69] or Girard's linear logic Gir87, where the logical rules manipulate only the topmost connectives of the formulas. In Gug02, and several other related works (e.g., BT01, GS01, Brü03, Str03]), it is shown that deep inference gives rise to a number of interesting proof theoretical properties for the logical systems formalized in the calculus of structures that are not observable in systems without deep inference. However, if one is concerned only with provability of logical expressions, deep inference might seem to introduce unnecessary non-determinism into proof search (although this problem has been partly addressed in Gug02, using a technique called splitting). The work presented in this paper had started with the conjecture that deep inference is not necessary for provability;

2000 ACM Subject Classification: F.4.1.

Key words and phrases: proof theory, deep inference, sequent calculus, calculus of structures, noncommutative logics.

A major part of this work was done when the author was at Fakultät Informatik, TU Dresden. The author received support from INRIA Lorraine (LORIA) during the completion of this work.

(C) A. Tiu (c) Creative Commons 
a conjecture which turns out to be wrong. We present a counterexample, a class of logical expressions in System BV Gug02, an extension of multiplicative linear logic with a self-dual non-commutative connective, which is provable only if there is no fixed bound to the depth of the applicability of the rules in BV, and hence rule out any candidate "shallow system" for BV.

The main result of this paper can be seen as a first technical argument to the claim that deep inference is a non-trivial extension to the traditional sequent calculus, in the sense that it allows for a simple formulation of a logic, which does not admit any straightforward formulation in sequent calculus without deep inference. By "sequent calculus without deep inference" we have in mind sequent systems with list-like contexts and whose logical rules manipulate only the topmost connectives. We regard such systems as a subset of shallow systems, the precise definition of which is given in Section [6. There are calculi other than the calculus of structures which also employ some sort of deep inference, notably, the logic of bunched implication OP99] and the display calculus Bel82, where the context of the sequent is a tree-like structure and inference rules can access any part of the tree. But there has been so far no technical analyses in those calculi concerning the necessity of deep inference.

We hope also to shed light on the known open problem of the sequentialization of Retoré's Pomset logic Ret97]. Pomset logic is an extension of multiplicative linear logic with a self-dual non-commutative connective. It is presented in the calculus of proofnets [Gir87, and there has to date been no known sequentialization theorem yet. The logic BV and Pomset logic are closely related; in fact, there is a sound translation from BV to Pomset logic preserving provability Str03. We conjecture that they are actually the same logic. The result on the necessity of deep-inference of BV therefore explains to some extent the difficulty in the sequentialization of Pomset logic.

This paper is organised as follows. In Section 2 we define structures, the analog to sequents in sequent calculus. This is then followed by a presentation of System BV in Section 3. Section 4 gives an overview of the main idea behind the proof of the necessity of deep inference, that is, the construction of a class of provable structures in BV which serve as the counterexample that rules out any candidate shallow system for BV. The formal proof for the necessity of deep inference relies on a graph representation of structures, called the relation webs Gug02, which is reviewed in Section 5. Section 6 gives a technical definition of shallow systems. In Section 7 we prove formally the necessity of deep-inference for System $\mathrm{BV}$ : for every candidate shallow system for BV, there is a structure provable in BV but not provable in that candidate system. Section 8 concludes the paper.

\section{Structures}

The logical expressions in the calculus of structures are called structures. A structure can be seen as representing a class of logical formulas, where each logical connective corresponds to a unique structure-forming relation, or structural relation. This is similar to the reading of sequents in sequent calculus: a (one-sided) sequent $\vdash A, B$ in linear logic can be seen as representing both the formulas $A \ngtr B$ and $B \ngtr A$, interpreting the ',' (comma) in the sequent as 8 . However, unlike sequent calculus, since each connective is assigned its own structural relation, the distinction between connectives and strutural relations are not particularly relevant if we consider only the combinatorial properties of the logical systems in the calculus of structures (e.g., permutability, cut-elimination, etc.). We are therefore 


$$
\begin{gathered}
\text { Associativity } \\
{[\vec{R},[\vec{T}]]=[\vec{R}, \vec{T}]} \\
(\vec{R},(\vec{T}))=(\vec{R}, \vec{T}) \\
\langle\vec{R} ;\langle\vec{T}\rangle ; \vec{U}\rangle=\langle\vec{R} ; \vec{T} ; \vec{U}\rangle \\
\text { Unit } \\
{[\circ, \vec{R}]=[\vec{R}]} \\
(\circ, \vec{R})=(\vec{R}) \\
\langle\circ ; \vec{R}\rangle=\langle\vec{R} ; \circ\rangle=\langle\vec{R}\rangle \\
\text { Singleton } \\
{[R]=(R)=\langle R\rangle=R}
\end{gathered}
$$

Commutativity

$$
\begin{aligned}
& {[\vec{R}, \vec{T}]=[\vec{T}, \vec{R}]} \\
& (\vec{R}, \vec{T})=(\vec{T}, \vec{R})
\end{aligned}
$$

\section{Negation}

$$
\begin{aligned}
& \begin{aligned}
& \frac{\overline{\mathrm{o}}}{\left\lceil R_{1}, \ldots, R_{h}\right\rceil}=\circ \\
& \frac{\left(R_{1}, \ldots, R_{h}\right)}{\left(R_{1}, \ldots, \bar{R}_{h}\right)}
\end{aligned} \\
& \underline{\left(R_{1}, \ldots, R_{h}\right)}=\left[\bar{R}_{1}, \ldots, \bar{R}_{h}\right] \\
& \frac{\left\langle R_{1} ; \ldots ; R_{h}\right\rangle}{\left\langle R_{1}\right.}=\left\langle\bar{R}_{1} ; \ldots ; \bar{R}_{h}\right\rangle \\
& \bar{R}=R
\end{aligned}
$$

\section{Contextual Closure}

if $R=T$ then $S\{R\}=S\{T\}$

Figure 1: Syntactic equivalence $=$

contend with having just structures as the only syntactical expressions of the logical systems in calculus of structures. The class of structures we are interested in is the following.

Definition 2.1. There are infinitely many positive atoms and negative atoms. Atoms, positive or negative, are denoted by $a, b, \ldots$ Structures are denoted by $S, P, Q, R, T, U$ and $V$. The structures of the language BV are generated by

$$
S::=a|\circ|[\underbrace{S, \ldots, S}_{>0}]|(\underbrace{S, \ldots, S}_{>0})|\langle\underbrace{S ; \ldots ; S}_{>0}\rangle \mid \bar{S}
$$

where $\circ$, the unit, is not an atom; $\left[S_{1}, \ldots, S_{h}\right]$ is a par structure, $\left(S_{1}, \ldots, S_{h}\right)$ is a copar structure and $\left\langle S_{1} ; \ldots ; S_{h}\right\rangle$ is a seq structure; $\bar{S}$ is the negation of the structure $S$. Structures with a hole not occuring in the scope of a negation are denoted by $S\{\}$. The structure $R$ is a substructure of $S\{R\}$, and $S\{\}$ is its context.

The par and co-par structural relations correspond to the 8 and the $\otimes$ connectives in linear logic Gir87, while the seq-relation is a self-dual non-commutative connective. We often omit the curly braces in a structure context in cases where structural parentheses fill the hole exactly: for example, we shall write $S[R, T]$ to mean $S\{[R, T]\}$.

Definition 2.2. The structures of the language BV are equivalent modulo the relation $=$, defined in Figure 1. There, $\vec{R}, \vec{T}$ and $\vec{U}$ stand for finite, non-empty sequences of structures (sequences may contain ',' or ';' separators as appropriate in the context). A structure $S$ is in normal form when either $S=\circ$ or there is no unit o appearing in it and the only negated structures appearing in it are atoms. Similarly, a structure context $S\{\}$ is in normal form when there is no unit $\circ$ appearing in it and the only negated structures appearing in it are atoms. Given a structure $S$, we talk about the atom occurrences of $S$ when we consider all the atoms appearing in $S$ as distinct. Therefore, in the structure $\langle a ; a\rangle$ there are two different occurrences of the atom $a$. The set of all the atom occurrences in $S$ is denoted with occ $S$.

We shall now define precisely the depth of a structure context. This definition will come handy later when proving various properties related to the depth of inference rules. 


$$
\text { ai } \frac{S\{\circ\}}{S[a, \bar{a}]} \quad \text { ai } \frac{S(a, \bar{a})}{S\{\circ\}} \quad \text { q } \downarrow \frac{S\left\langle[R, T] ;\left[R^{\prime}, T^{\prime}\right]\right\rangle}{S\left[\left\langle R ; R^{\prime}\right\rangle,\left\langle T ; T^{\prime}\right\rangle\right]} \quad \text { q } \uparrow \frac{S\left(\langle R ; T\rangle,\left\langle R^{\prime} ; T^{\prime}\right\rangle\right)}{S\left\langle\left(R, R^{\prime}\right) ;\left(T, T^{\prime}\right)\right\rangle} \quad \mathrm{s} \frac{S\left([R, T], R^{\prime}\right)}{S\left[\left(R, R^{\prime}\right), T\right]}
$$

Figure 2: System SBV

Note that since structures and structure contexts are considered modulo associativity and commutativity (for par and co-par), the depth of a structure context is also considered modulo these equalities. In its normal form, a structure can be viewed as a finitely branching tree. A structure context is then a particular tree with a hole \{\} as a leaf. The depth is measured as the length of the branch ending with \{\} . The formal definition is as follows.

Definition 2.3. Let $S\{\}$ be a normal structure context. The depth of $S\{\}$ is defined as follows:

(1) $\operatorname{depth}\{\}=0$,

(2) depth $\left[S_{1}, S_{2}\{\}\right]= \begin{cases}\operatorname{depth} S_{2}\{\}, & \text { if } S_{2}\{\}=\left[S_{2}^{\prime}, S_{2}^{\prime \prime}\{\}\right], \\ \operatorname{depth} S_{2}\{\}+1, & \text { otherwise, }\end{cases}$

(3) depth $\left(S_{1}, S_{2}\{\}\right)= \begin{cases}\operatorname{depth} S_{2}\{\}, & \text { if } S_{2}\{\}=\left(S_{2}^{\prime}, S_{2}^{\prime \prime}\{\}\right), \\ \operatorname{depth} S_{2}\{\}+1, & \text { otherwise, }\end{cases}$

(4) depth $\left\langle S_{1} ; S_{2}\{\}\right\rangle= \begin{cases}\operatorname{depth} S_{2}\{\}, & \text { if } S_{2}\{\}=\left\langle S_{2}^{\prime} ; S_{2}^{\prime \prime}\{\}\right\rangle \\ \operatorname{depth} S_{2}\{\}+1, & \text { or } S_{2}\{\}=\left\langle S_{2}^{\prime}\{\} ; S_{2}^{\prime \prime}\right\rangle,\end{cases}$

(5) depth $\left\langle S_{1}\{\} ; S_{2}\right\rangle= \begin{cases}\operatorname{depth} S_{1}\{\}, & \text { if } S_{1}\{\}=\left\langle S_{1}^{\prime} ; S_{1}^{\prime \prime}\{\}\right\rangle \\ \operatorname{depth} S_{1}\{\}+1, & \text { or } S_{1}\{\}=\left\langle S_{1}^{\prime}\{\} ; S_{1}^{\prime \prime}\right\rangle,\end{cases}$

For example, the structure context $[a, b,\{\}]$ has depth 1 and $[\langle\{\} ; c\rangle,\langle b ; c\rangle]$ has depth 2. A structure $R$ is said to occur at depth $n$ in $S\{R\}$ if $n$ is the depth of $S\{\}$.

\section{System BV}

In this section, we review some key concepts and terminologies in the calculus of structures and present System BV.

Recall that we associate to each connective a structural relation. This has a consequence on the shape of inference rules in the calculus of structures: there is basically no need for branching inference rules, i.e., inference rules with more than one premise. Derivations in the calculus of structures are sequences of inference rules, instead of tree-shape structures. Consider for example the following tensor rule of linear logic in sequent calculus:

$$
\otimes \frac{\vdash A, \Phi \quad \vdash B, \Psi}{\vdash A \otimes B, \Phi, \Psi}
$$

In the calculus of structure, we would present the rule as something like

$$
\otimes \frac{([A, \Phi],[B, \Psi])}{[(A, B), \Phi, \Psi]}
$$


Here we use the par structural relation to interpret $>$ and the co-par to interpret $\otimes$. This simulation of the tensor rule in the calculus of structures can be generalized to arbitrary onesided sequent systems. This idea has been used to simulate several known sequent systems inside the calculus of structures (see e.g., BT01, Str03), through which the cut-elimination results are obtained for the corresponding systems in the calculus of structures.

Another important feature of the calculus of structures is that it allows deep inference. Inference rules can be applied to any substructure of a given structure. Thus, inference rules in the calculus of structures can be seen as rewrite rules on structures. This is in contrast to traditional sequent systems where introduction rules are usually applied to the topmost connectives of formulas. Typical rules in the calculus of structures have the form:

$$
\rho \frac{S\{T\}}{S\{R\}}
$$

where $\rho$ is the name of the rule, $R$ and $T$ are structures and $S\{\}$ is a structure context. Common to all systems already studied in the calculus of structures are the interaction rules:

$$
\text { i } \frac{S\{\circ\}}{S[R, \bar{R}]} \quad \text { and } \quad \text { i } \frac{S(R, \bar{R})}{S\{\circ\}}
$$

which correspond to the identity rule and the cut rule in sequent calculus, respectively.

We shall now define precisely System BV with its notions of inference rules, derivations and proofs.

Definition 3.1. An inference rule is a scheme $\rho \frac{T}{R}$, where $\rho$ is the name of the rule, $T$ is its premise and $R$ is its conclusion. Rule names are denoted by $\rho$ and $\pi$. A formal system, denoted by $\mathscr{S}$, is a set of rules. A derivation in a system $\mathscr{S}$ is a finite chain of instances of rules of $\mathscr{S}$, and is denoted by $\Delta$. A derivation can consist of just one structure. The topmost structure in a derivation is called its premise; the lowest structure is called conclusion. A derivation $\Delta$ whose premise and conclusion are $T$ and $R$, and whose rules are in $\mathscr{S}$ is denoted by $\underset{R}{\Delta} \|_{R}$. An instance of a rule $\rho \frac{T}{R}$ is called non-trivial if $R \neq T$, otherwise it is trivial.

Definition 3.2. System SBV GS01] is shown in Figure 2, The rules in SBV are of the form $\rho \frac{S\{T\}}{S\{R\}}$. The structure $R$ is called redex and the structure $T$ is called contractum.

The rules ai $\downarrow$ and ai $\uparrow$ are the atomic versions of the more general interaction rules i $\downarrow$ and $i \uparrow$. The non-atomic interaction rules can be reduced to the atomic ones, as shown in GS01. System SBV consists of a down fragment $\{\mathrm{ai} \downarrow, \mathrm{s}, \mathrm{q} \downarrow\}$ and an up fragment $\{\mathrm{ai} \uparrow, \mathrm{s}, \mathrm{q} \uparrow\}$. The rules ai $\downarrow$ and $\mathrm{q} \uparrow$ in the up fragment are admissible (for proofs) as a consequence of the cut elimination theorem for this system Gug02. Therefore, it is enough to study the down fragment for its proof search properties. We need an extra rule, a logical axiom rule, to define proofs in SBV.

Definition 3.3. The down fragment of SBV, together with the rule $\circ \downarrow \frac{}{\circ}$, is called System BV (Figure 3). 


$$
\circ \downarrow \frac{1}{\circ} \quad \text { ai } \downarrow \frac{S\{\circ\}}{S[a, \bar{a}]} \quad \mathrm{s} \frac{S\left([R, T], R^{\prime}\right)}{S\left[\left(R, R^{\prime}\right), T\right]} \quad \mathrm{q} \downarrow \frac{S\left\langle[R, T] ;\left[R^{\prime}, T^{\prime}\right]\right\rangle}{S\left[\left\langle R ; R^{\prime}\right\rangle,\left\langle T ; T^{\prime}\right\rangle\right]}
$$

Figure 3: System BV

Definition 3.4. A proof, denoted by $\Pi$, is a derivation whose top is an instance of $\circ \downarrow$. A system $\mathscr{S}$ proves $R$ if there is in $\mathscr{S}$ a proof $\Pi$ whose conclusion is $R$, written $\begin{array}{r}\Pi \pi \mathscr{S} \\ R\end{array}$. The length of a proof $\Pi$ is the number of occurrences of inference rules in $\Pi$. Two systems are equivalent if they prove the same structures.

Given a provable structure in BV, we can remove all the dual instances of atoms, except maybe for some pairs, and get another provable structure. In this way, we can see whether there are certain local properties obeyed by all provable structures. This will be useful later when proving the necessity of deep inference (see Section [7).

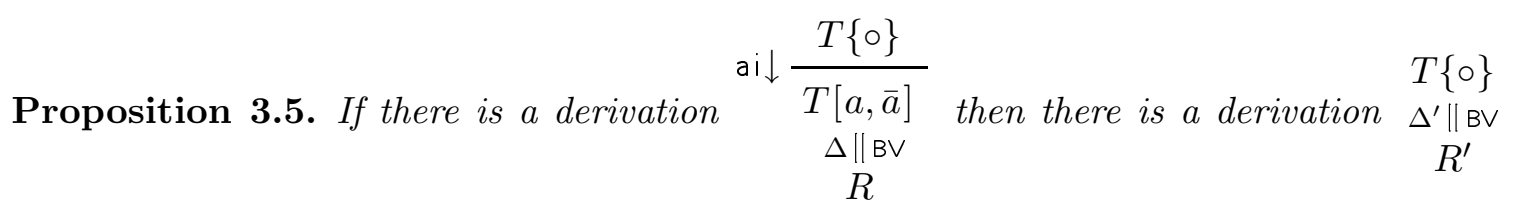

where $R^{\prime}$ and $\Delta^{\prime}$ are obtained from $R$ and $\Delta$, respectively, by replacing the atom occurrences $a$ and $\bar{a}$ with units.

Proof. By simple induction on the length of $\Delta$.

\section{Overview of the Counterexample}

The counterexample is based on the structure

$$
[\langle[a, b] ; c\rangle,\langle\bar{a} ;[\bar{b}, \bar{c}]\rangle],
$$

which we shall refer to as $S_{0}$. In order to prove the structure, all dual pairs of atoms must be brought into the same par substructures so that the interaction rule ai $\downarrow$ can be applied. It is quite obvious that more than one application of inference rules in BV is needed to bring the dual pairs of atoms into the same par substructures. For instance, to bring $a$ and $\bar{a}$ into the same substructure (via the $q \downarrow$ rule), the par substructure $[a, b]$ needs to be changed to $\langle a ; b\rangle$ first. The same observation applies to the substructure $[\bar{b}, \bar{c}]$ : it must be changed to $\langle\bar{b} ; \bar{c}\rangle$ first before $c$ can be brought into the same par substructure with $\bar{c}$. It can be shown that $S_{0}$ has the following property: all of its proofs must begin with (reading the proofs bottom up) either the substructure $[a, b]$ or $[\bar{b}, \bar{c}]$ as the redex. A proof of $S_{0}$ is given in Figure 4 where the first redex is the substructure $[a, b]$ (modulo structure equality). The underlined substructures in the proof are redexes of the rules. The equality sign in the rules indicates applications of structure equalities. There is another proof of $S_{0}$ which is not shown and which starts with $[\bar{b}, \bar{c}]$ as the redex. The substructures $[a, b]$ and $[\bar{b}, \bar{c}]$ occur at depth 2 in $S_{0}$. Therefore this structure is provable in a given candidate shallow system for BV only if the proof system allows for rules which apply beyond depth 2 in the structure (the notion of the depth of inference rules will be made precise later). 


$$
\begin{aligned}
& \circ \downarrow- \\
& =\frac{0}{\langle 0 ; 0\rangle}
\end{aligned}
$$

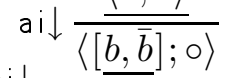

$$
\begin{aligned}
& \text { ai } \downarrow \frac{\langle[\bar{b} \overline{\bar{b}]} ; \underline{[c, \bar{c}]\rangle}}{\langle[\bar{b}}
\end{aligned}
$$

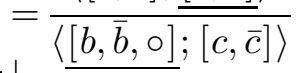

$$
\begin{aligned}
& \mathrm{q} \downarrow \frac{\overline{\langle[b, \bar{b}] ; c\rangle,\langle\circ ; \bar{c}\rangle]}}{[\langle b, \mathrm{~b}}
\end{aligned}
$$

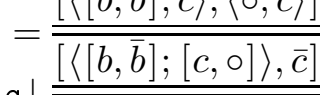

$$
\begin{aligned}
& \mathrm{q} \downarrow \overline{\underline{\underline{\langle b ; c\rangle,\langle\bar{b} ; \circ\rangle, \bar{c}]}}} \\
& =\frac{[\langle b ; c\rangle, \underline{\bar{b}}, \bar{c}]}{\langle\circ ;[\langle b ; c\rangle, \bar{b}, \bar{c}]\rangle} \\
& \text { ai } \frac{}{\langle[a, \bar{a}] ;[\langle b ; c\rangle, \bar{b}, \bar{c}]\rangle} \\
& \mathrm{q} \downarrow \overline{[\langle a ; b ; c\rangle,\langle\bar{a} ;[\bar{b}, \bar{c}]\rangle]} \\
& \begin{array}{l}
=\frac{\underline{[\langle\langle[a, \circ] ;[\circ, b]\rangle ; c\rangle,\langle\bar{a} ;[\bar{b}, \bar{c}]\rangle]}}{\mathrm{q}} \\
=\frac{[\langle[\langle a ; \circ\rangle,\langle\circ ; b\rangle] ; c\rangle,\langle\bar{a} ;[\bar{b}, \bar{c}]\rangle]}{[\langle[a, b] ; c\rangle,\langle\bar{a} ;[\bar{b}, \bar{c}]\rangle]} .
\end{array}
\end{aligned}
$$

Figure 4: A proof of the $S_{0}$ structure

Another interesting property of the $S_{0}$ structure is that it can be superimposed with itself, forming a bigger structure such that its proofs must begin with an innermost par substructure as the redex. We illustrate here how such structures can be constructed, the formal definition will be given in Section 7 . Consider the following "parameterized' $S_{0}$ :

$$
[\langle[a, b, R] ; c\rangle,\langle\bar{a} ;[\bar{b}, \bar{c}, T]\rangle] \text {. }
$$

We denote with $S_{0}\{U\}\{V\}$ the above structure with $U$ replacing $R$ and $V$ replacing T. The following structure is a superimposition of three $S_{0}$ structures, which we call $S_{1}$ :

$$
\left[\left\langle S_{0}\left\{a_{1}\right\}\left\{b_{1}\right\} ; c_{1}\right\rangle,\left\langle\bar{a}_{1} ; S_{0}\left\{\bar{b}_{1}\right\}\left\{\bar{c}_{1}\right\}\right\rangle\right] .
$$

A proof of $S_{1}$ can be constructed from the schematic derivation of the parameterized $S_{0}$ in Figure 5. That is, we apply the derivation scheme to the substructures $S_{0}\left\{a_{1}\right\}\left\{b_{1}\right\}$ and $S_{0}\left\{\bar{b}_{1}\right\}\left\{\bar{c}_{1}\right\}$, yielding the structure $\left[\left\langle\left[a_{1}, b_{1}\right] ; c_{1}\right\rangle,\left\langle\bar{a}_{1} ;\left[\bar{b}_{1}, \bar{c}_{1}\right]\right\rangle\right]$. The latter is an $S_{0}$ structure (modulo renaming of atoms) and is therefore provable. Again as with $S_{0}$, it can be shown that any proof of $S_{1}$ must begin with an innermost par substructure as the redex. In this case, the depth of the redex is 4 . The $S_{1}$ structure can be further superimposed with other $S_{0}$ structures to produce bigger structures with the same property. A recursive procedure to produce such structures is given in Section 7 . As we shall see later, given any $n$, this procedure generates a provable structure with the property that its proofs must begin with a redex at depth $2 n$. Thus given any candidate shallow system for BV, a provable structure can be generated such that its proofs must start at a depth that is beyond the depth of the rules in that particular shallow system, and hence cannot be proved in the system. 


$$
\begin{aligned}
& =\frac{[R, T]}{\text { ai } \downarrow \frac{\langle[\circ, R, T] ; \circ\rangle}{\langle[b, \bar{b}, R, T] ; \circ\rangle}} \\
& =\frac{\left\langle i \downarrow \frac{\langle\bar{b}, R, T] ; \underline{[c, \bar{c}]}\rangle}{\langle[\bar{b}, \bar{b}, R, T, \circ] ;[c, \bar{c}]\rangle}\right.}{\underline{\langle[b, \bar{b}, R, T] ; c\rangle,\langle\circ: c)]}} \\
& \mathrm{q} \downarrow \frac{\overline{[\langle[b, \bar{b}, R, T] ; c\rangle,\langle\circ ; \bar{c}\rangle]}}{[\langle[b, R, b, T] ;(c, \circ]\rangle, \bar{c}]} \\
& =\frac{\underline{[\langle[b, R, \bar{b}, T] ;[c, \circ]\rangle, \bar{c}]}}{\underline{[\langle b, R] ; c\rangle,\langle[\bar{b}, T] ; \circ\rangle, \bar{c}]}}
\end{aligned}
$$

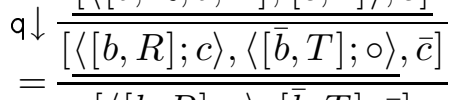

$$
\begin{aligned}
& =\frac{[\langle[b, R] ; c\rangle,[\bar{b}, T], \bar{c}]}{\langle\circ ;[\langle[b, R] ; c\rangle, \bar{b}, \bar{c}, T]\rangle} \\
& \text { ai } \frac{}{\langle[\overline{[a, \bar{a}] ;} ;\langle[b, R] ; c\rangle, \bar{b}, \bar{c}, T]\rangle} \\
& \mathrm{q} \downarrow \frac{\overline{\langle a ;[b, R] ; c\rangle,\langle\bar{a} ;[\bar{b}, \bar{c}, T]\rangle]}}{\overline{[a, j}} \\
& =\frac{\overline{[\langle\langle a, \circ] ;[\circ, b, R]\rangle ; c\rangle,\langle\bar{a} ;[\bar{b}, \bar{c}, T]\rangle]}}{\left[\left\langle{ }^{\prime}[\langle a ; o\rangle,\langle o ; b, R]\rangle ; c\right\rangle,\langle\bar{a} ;[\bar{b}, \bar{c}, T]\rangle\right]} \\
& \mathrm{q} \downarrow \frac{\overline{[\langle[\langle a ; \circ\rangle,\langle\circ ;[b, R]\rangle] ; c\rangle,\langle\bar{a} ;[\bar{b}, \bar{c}, T]\rangle]}}{[\underline{[\langle a, b, R] ; c\rangle,\langle\bar{a} ;[\bar{b}, \bar{c}, T]\rangle]}} .
\end{aligned}
$$

Figure 5: A derivation for the parameterized $S_{0}$

\section{Characterisation of Structures}

In this section we consider a different representation for structures, a special kind of graph called relation webs Gug02 (originally called traces Gug99]), which is a generalisation of series-parallel orders [Möh89]. In this representation, structures can be characterized by the absence of certain forbidden configurations in their relation webs. This characterisation is useful when we want to study general properties of inference rules. This section reviews the definitions and the main results concerning relation webs already presented in Gug99, Gug02.

Let $S$ be a structure in normal form. The structural relations $\triangleleft_{S}(s e q), \triangleright_{S}(c o-s e q), \downarrow_{S}$ (par) and $\uparrow_{S}\left(\right.$ co-par) are defined as the smallest sets such that $\triangleleft_{S}, \triangleright_{S}, \downarrow_{S}, \uparrow_{S} \subset$ (occ $\left.S\right) \times$ (occ $S$ ) and, for every $S^{\prime}\{\}, U$ and $V$ and for every $a$ in $U$ and $b$ in $V$, the following hold:

(1) if $S=S^{\prime}\langle U ; V\rangle$ then $a \triangleleft_{S} b$ and $b \triangleright_{S} a$;

(2) if $S=S^{\prime}[U, V]$ then $a \downarrow_{S} b$;

(3) if $S=S^{\prime}(U, V)$ then $a \uparrow_{S} b$.

To a structure that is not in normal form we associate the structural relations obtained from any of its normal forms. The quadruple ( $\operatorname{ccc} S, \triangleleft_{S}, \downarrow_{S}, \uparrow_{S}$ ) is called the relation web of $S$, written $\mathrm{w}_{S}$. We shall omit the subscripts in $\triangleleft_{S}, \triangleright_{S}, \downarrow_{S}$ and $\uparrow_{S}$ when it is clear from context which structure we refer to. Given two sets of atom occurrences $\mu$ and $\nu$, we write $\mu \triangleleft \nu, \mu \triangleright \nu, \mu \downarrow \nu$ and $\mu \uparrow \nu$ to indicate situations where, for every $a$ in $\mu$ and for every $b$ in $\nu$, they hold, respectively, $a \triangleleft b, a \triangleright b, a \downarrow b$ and $a \uparrow b$. For example, in $(\langle a, \bar{b}\rangle,[\bar{c}, d])$ we have the following relations: $a \triangleleft \bar{b}, a \uparrow \bar{c}, a \uparrow d, \bar{b} \triangleright a, \bar{b} \uparrow \bar{c}, \bar{b} \uparrow d, \bar{c} \uparrow a, \bar{c} \uparrow \bar{b}, \bar{c} \downarrow d, d \uparrow a$, $d \uparrow \bar{b}, d \downarrow \bar{c}$. 


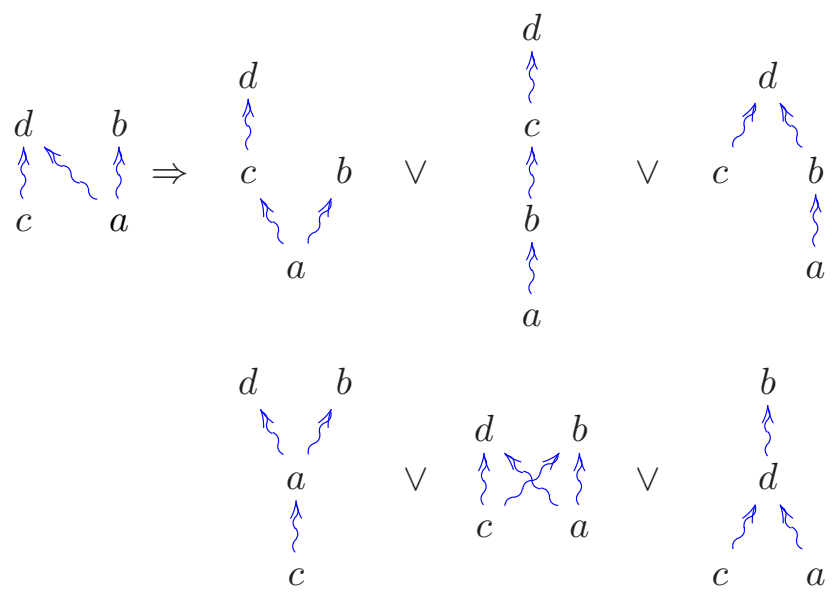

Figure 6: Square property for $\triangleleft$

The following proposition states that all the atoms in a sub-structure are in the same structural relations with respect to each of the atoms surrounding them:

Proposition 5.1. Gug02 Given a structure $S\{R\}$ and two atom occurrences a in $S\{\}$ and $b$ in $R$, if $a \triangleleft b$ (respectively, $a \triangleright b, a \downarrow b, a \uparrow b$ ) then $a \triangleleft c$ (respectively, $a \triangleright c, a \downarrow c$, $a \uparrow c)$ for all the atom occurrences $c$ in $R$.

Theorem 5.2. Gug02 Given $S$ and its associated structural relations $\triangleleft, \triangleright, \downarrow$ and $\uparrow$, the following properties hold, where $a, b, c$ and $d$ are distinct atom occurrences in $S$ :

$\mathrm{s}_{1}$ : None of $\triangleleft, \triangleright, \downarrow$ and $\uparrow$ is reflexive: $\neg(a \triangleleft a), \neg(a \triangleright a), \neg(a \downarrow a), \neg(a \uparrow a)$.

$\mathrm{s}_{2}$ : One and only one among $a \triangleleft b, a \triangleright b, a \downarrow b$ and $a \uparrow b$ holds.

$\mathrm{s}_{3}$ : The relations $\triangleleft$ and $\triangleright$ are mutually inverse: $a \triangleleft b \Leftrightarrow b \triangleright a$.

$\mathrm{s}_{4}$ : The relations $\triangleleft$ and $\triangleright$ are transitive: $(a \triangleleft b) \wedge(b \triangleleft c) \Rightarrow a \triangleleft c$ and $(a \triangleright b) \wedge(b \triangleright$ c) $\Rightarrow a \triangleright c$.

$\mathrm{s}_{5}:$ The relations $\downarrow$ and $\uparrow$ are symmetric: $a \downarrow b \Leftrightarrow b \downarrow a$ and $a \uparrow b \Leftrightarrow b \uparrow a$.

$\mathrm{s}_{6}$ : Triangular property: for $\sigma_{1}, \sigma_{2}, \sigma_{3} \in\{\triangleleft \cup \triangleright, \downarrow, \uparrow\}$, it holds

$$
\left(a \sigma_{1} b\right) \wedge\left(b \sigma_{2} c\right) \wedge\left(c \sigma_{3} a\right) \Rightarrow\left(\sigma_{1}=\sigma_{2}\right) \vee\left(\sigma_{2}=\sigma_{3}\right) \vee\left(\sigma_{3}=\sigma_{1}\right) .
$$

$\mathrm{s}_{7}$ : Square property:

$$
\begin{array}{llll}
s_{7}^{\triangleleft} & (a \triangleleft b) \wedge(a \triangleleft d) \wedge(c \triangleleft d) \Rightarrow & & (a \triangleleft c) \vee(b \triangleleft c) \vee(b \triangleleft d) \vee \\
& & (c \triangleleft a) \vee(c \triangleleft b) \vee(d \triangleleft b), \\
s_{7}^{\downarrow} & (a \downarrow b) \wedge(a \downarrow d) \wedge(c \downarrow d) \Rightarrow & & (a \downarrow c) \vee(b \downarrow c) \vee(b \downarrow d), \\
s_{7}^{\uparrow} & (a \uparrow b) \wedge(a \uparrow d) \wedge(c \uparrow d) \Rightarrow & & (a \uparrow c) \vee(b \uparrow c) \vee(b \uparrow d) .
\end{array}
$$

Proof. See Gug02.

It is convenient to use graphical notations when reasoning about relation webs. We use the following drawings

$$
a \leadsto b, a \ll b \quad, a-b \text { and } a \sim b
$$




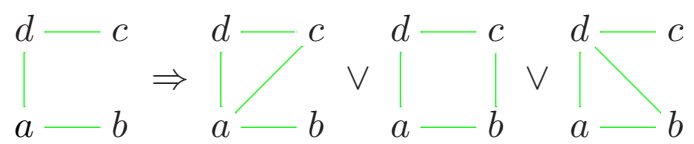

Figure 7: Square property for $\downarrow$

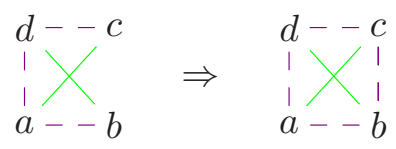

Figure 8: Inverse square property for $\downarrow$

to represent the strutural relations: $a \triangleleft b$ (and $b \triangleright a$ ), $a \triangleleft b$ or $a \triangleright b, a \downarrow b$ and $a \uparrow b$, respectively. Dashed arrows represent negations of structural relations, e.g.,

$$
a---b
$$

means that $\neg(a \downarrow b)$.

Theorem 5.2 tells us that certain configurations are absent in relation webs. The most interesting ones are those that derive from $\mathrm{s}_{6}$ (the triangular property) and $\mathrm{s}_{7}$ (the square property). The triangular property says that the following configuration is absent in relation webs:

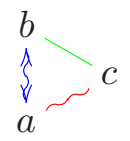

For a structure with three atom occurrences, it is easy to check that the above configuration is absent: simply check all possible structures that can be formed from the three atoms. For the more general case, one needs to consider the cases where the atoms are nested inside other substructures, e.g., as in $[U\{a\},\langle V\{b\} ; W\{c\}\rangle]$. Induction on structures is needed in this case (see Gug02 for details).

The square property for $\triangleleft$ and $\downarrow$ are presented graphically in Figure 6 and Figure 7 respectively. The square property for $\uparrow$ is similar to the one for $\downarrow$. From the square property, we can infer, for example, that there is no structure containing the following configuration in its relation web

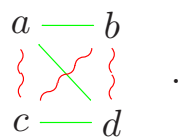

A useful corollary of Theorem 5.2 is that the square property holds also for some negated relations.

Corollary 5.3. Inverse square property. Given $S$ and its associated structural relations $\triangleleft$, $\triangleright, \downarrow$ and $\uparrow$, the following properties hold, where $a, b, c$ and $d$ are distinct atom occurrences in $S$ :

(1) if $\neg(a \downarrow b), \neg(a \downarrow d), \neg(c \downarrow d), a \downarrow c$ and $b \downarrow d$ then $\neg(b \downarrow c)$,

(2) if $\neg(a \uparrow b), \neg(a \uparrow d), \neg(c \uparrow d), a \uparrow c$ and $b \uparrow d$ then $\neg(b \uparrow c)$. 
Proof. We show the case for the $\downarrow$ relation, the other case is proved analogously. Suppose that $\neg(a \downarrow b), \neg(a \downarrow d), \neg(c \downarrow d), a \downarrow c$ and $b \downarrow d$ hold, but $b \downarrow c$. Then applying the square property for $\downarrow$ in Theorem 5.2 it must be the case that one of the following relations holds: $a \downarrow b, a \downarrow d, c \downarrow d$. But either one of them would result in a contradiction with the assumptions.

Figure 8 shows the graphical representation of the inverse square property for the $\downarrow$ relation. Note that the inverse square property for the $\triangleleft$ relation, i.e., "if $\neg(a \triangleleft b), \neg(a \triangleleft d)$, $\neg(c \triangleleft d), a \triangleleft c$ and $b \triangleleft d$ then $\neg(b \triangleleft c)$ " does not hold. Consider for instance the structure $\langle b ;[\langle a ; c\rangle, d]\rangle$. Its relation web is the following:

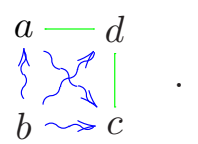

We have in the web $\neg(a \triangleleft b), \neg(a \triangleleft d), \neg(c \triangleleft d), a \triangleleft c$ and $b \triangleleft d$ but $b \triangleleft c$.

The conditions $s_{1}-s_{7}$ in Theorem 5.2 turn out to be sufficient for characterizing structures, that is, every web candidate (i.e., a graph with edges labelled with the relations $\triangleleft$, $\downarrow$ and $\uparrow$ ) which satisfies the conditions is a relation web (hence a structure). This is proved in Gug02 where a non-deterministic algorithm is given to decide whether a web candidate is a relation web. We shall not go into the details of the general algorithm and its correctness proof since they are not needed in establishing the main results of this paper. We illustrate here briefly how one can recover a structure from a given relation web using the algorithm. The algorithm works by identifying substructures in the relation web, starting from the smallest ones. At each step of the algorithm, partitions are formed on the web such that each partition corresponds to a valid (sub)structure. Given a relation web $w_{S}$, the steps to recover the structure corresponding to the web is as follows:

First step: Form a partition $\{a\}$ for each atom occurrence $a$ in the web.

Iterative step: Let $\mu$ and $\nu$ be two distinct partitions (which are valid substructures) such that all atom occurrences in $\mu$ and $\nu$ are in the same relations, and for all $a \in \mu, b \in \nu$ and $c \in \operatorname{occ} S \backslash(\mu \cup \nu), a \triangleleft c$ (respectively, $a \downarrow c$ and $a \uparrow c$ ) iff $b \triangleleft c$ (respectively, $b \downarrow c$ and $b \uparrow c$ ). Merge the partitions $\mu$ and $\nu$. Let $U$ be the corresponding structure of $\mu$ and $V$ be the corresponding structure of $\nu$. If $\mu$ and $\nu$ are related by $\downarrow$ (respectively, $\triangleleft$ and $\uparrow$ ) then the corresponding structure for the merged partition is $[U, V]$ (respectively, $\langle U ; V\rangle$ and $(U, V)$ ). Repeat this step until no merging is possible. The structure corresponding to the remaining partition (which should be the relation web $w_{S}$ itself) is the structure of the relation web.

Consider for instance the following web:

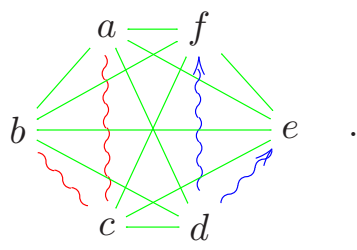

The intermediate partitions produced in the execution of the above algorithm is as follows, where we represent each partition with its corresponding structure:

(1) $a, b, c, d, e, f$.

(2) $[a, b], c, d, e, f$. 
(3) $[a, b], c, d,[e, f]$.

(4) $([a, b], c), d,[e, f]$.

(5) $([a, b], c),\langle d ;[e, f]\rangle$.

(6) $[([a, b], c),\langle d ;[e, f]\rangle]$.

The above relation web therefore corresponds to the structure $[([a, b], c),\langle d ;[e, f]\rangle]$.

The following theorem states that relation webs are a canonical representation of structures.

Theorem 5.4. Gug02 Two structures are equivalent if and only if they have the same relation web.

\section{Shallow Systems}

In the definition of shallow systems to follow, we shall try to be as general as possible but should also be careful to rule out certain inconsistent rules. An obvious case would be rules which would allow one to prove $A \ngtr B \multimap A \otimes B$, for example. Two conditions are enforced on the definition of candidate shallow systems: a shallow system should have a notion of cut and cut-elimination property, and further, the inference rules should respect a certain ordering of logical strength of structural relations. The latter can be seen as a sort of "subformula" property. We observe the following ordering on the structural relations: $\downarrow \prec \triangleleft \prec \uparrow$ and $\downarrow \prec \triangleright \prec \uparrow$.

Definition 6.1. Let $R$ and $T$ be two structures such that $R \neq T$ and occ $R=\operatorname{occ} T$. The relation $R \prec T$ holds if for all atom occurrences $a$ and $b$ in $R$, the following hold:

(1) if $a \downarrow_{T} b$ then $a \downarrow_{R} b$,

(2) if $a \uparrow_{T} b$ then either one of the following holds: $a \triangleleft_{R} b, a \triangleright_{R} b, a \uparrow_{R} b$ or $a \downarrow_{R} b$,

(3) if $a \triangleleft_{T} b$ then either $a \triangleleft_{R} b$ or $a \downarrow_{R} b$,

(4) if $a \triangleright_{T} b$ then either $a \triangleright_{R} b$ or $a \downarrow_{R} b$.

We shall now formalize what we mean by shallow inference rules and shallow systems. For this we define formally the notion of structure schemes, that is, structures containing variables. Structure scheme is then used in defining rule scheme and the depth of a rule scheme.

Definition 6.2. We enrich the language of structures in Definition 2.1 with a denumerable infinite set of structure variables. Structure variables are denoted with $A, B$ and $C$. The extended language of structures is defined as follows:

$$
S::=a|A| \circ|[\underbrace{S, \ldots, S}_{>0}]|(\underbrace{S, \ldots, S}_{>0})|\langle\underbrace{S ; \ldots ; S}_{>0}\rangle| \bar{S}
$$

where $a$ is an atom and $A$ is a structure variable. Structure variables representing atomic structures will also be denoted with $x, y$ and $z$. The definitions that apply to structures without variables apply also to structures with variables. In particular, occ $S$ now denotes the set of occurences of atoms and variables in the structure $S$. Definition 6.1 on the ordering of structures extends straightforwardly to structures with variables. The set of structure variables in $S$ is denoted by vars $S$. A ground structure is a structure that does not contain any variable. A structure with variables can be instantiated by replacing the variables with other structures. 
In the following definition of shallow systems, only the logical rules (i.e., the noninteraction rules) are required to be shallow, while the interaction rules are those of BV. Moreover, since the systems that are of interest will be those with cut-elimination property, we always refer to cut-free systems when we talk about shallow systems.

Definition 6.3. The depth of a structure $S$ is defined as follows:

$$
\operatorname{depth} S=\max \left\{\operatorname{depth} S^{\prime}\{\} \mid S^{\prime}\{R\}=S \text { and } R \in \operatorname{occ} S\right\}
$$

An inference rule $\rho \frac{T}{R}$ is shallow if $R$ and $T$ are non-unit structures such that $R \prec T$ and the variables in each of $R$ and $T$ are pairwise distinct. The depth of $\rho$ is defined as $\max (\operatorname{depth} R, \operatorname{depth} T)$.

Notice that the above definition of shallow inference rules excludes the interaction, contraction and weakening rules. An example of a shallow rule is the following rule

$$
\rho \frac{\left[A,\left([B, C], C^{\prime}\right)\right]}{\left[A, B,\left(C, C^{\prime}\right)\right]} .
$$

It is a shallow rule with depth 3 , which is the depth of the structure in the premise.

Definition 6.4. A shallow system $\mathscr{S}$ consists of the rules $\{i \downarrow, \circ \downarrow\}$ and a set of shallow rules $\mathscr{S}^{\prime}$ such that there exists an $n$, the depth of $\mathscr{S}$, such that for every rule $\rho \in \mathscr{S}^{\prime}, \operatorname{depth} \rho \leq n$.

In proving general properties of shallow rules, we shall use the relation webs semantics to characterize their operations on structures. An application of a non-interaction rule, seen at the relation webs level, modifies structural relations between atom occurrences in a structure. The square and triangular properties can be used to infer the effects on the overall structural relations in the structure. In addition, we also make use of the fact that there are some structural relations that are not modified by a shallow rule, if they happen to belong to a substructure that is nested deeper than the depth of the shallow rule. This is stated in the following lemma.

Definition 6.5. Let $\rho \frac{T}{R}$ be an instance of a rule $\rho$. The structural relation $a \sigma_{R} b$, where $a, b \in \operatorname{occ} R$ and $\sigma \in\{\downarrow, \triangleleft, \uparrow\}$, is preserved by $\rho$ if and only if $a, b \in \operatorname{occ} T$ and $a \sigma_{T} b$.

Lemma 6.6. Let $\rho \frac{T}{R}$ be an instance of a shallow rule $\rho$ of depth $n$ and $P$ be a structure occuring in $R$ at depth $m$. If $m>n$ then all the structural relations in $P$ are preserved by $\rho$.

Proof. The proof follows straightforwardly from the definition of shallow rules and relation webs.

\section{The Need for Deep Inference}

A main part of the proof of the necessity of deep-inference for BV is the construction of the class of provable structures which will serve as the counterexample to the completeness of any given shallow candidate system for BV. This class of structures is generated from the structure

$$
S_{0}=[\langle[a, b] ; c\rangle,\langle\bar{a} ;[\bar{b}, \bar{c}]\rangle] .
$$


It is done by merging recursively variants of $S_{0}$ with the innermost par-substructures of the original structure. The formal definition of this merging process is as follows.

Definition 7.1. A structure $R$ is called flat if all the structural relations between atom occurrences in $R$ are of the same type. The structure $\left[a_{1}, \ldots, a_{n}\right]$ is a flat par structure. Likewise, $\left(a_{1}, \ldots, a_{n}\right)$ and $\left\langle a_{1} ; \ldots ; a_{n}\right\rangle$ are a flat copar structure and a flat seq structure, respectively. A unit or an atomic structure can be seen as a flat par structure, flat copar structure or flat seq structure.

Definition 7.2. Let $\mathrm{C}$ be an infinite set of positive atoms and let $\mathrm{N}^{*}$ be the set of finite sequences of natural numbers. Elements of $\mathrm{N}^{*}$ are denoted by $u, v, w$ and $z$. Concatenation of two sequences of natural number $u$ and $v$ is denoted by $u . v$. The set of indexed atoms is defined as $\mathrm{C}_{\mathrm{N}^{*}}=\left\{a_{u} \mid a \in \mathrm{C}, u \in \mathrm{N}^{*}\right\}$. Two atoms with different names or indexes are considered different. The following functions generate a class of structures:

$$
\begin{aligned}
& \alpha_{0}(u, R, T)=\left[\left\langle\left[a_{u}, b_{u}, R\right] ; c_{u}\right\rangle,\left\langle\bar{a}_{u} ;\left[\bar{b}_{u}, \bar{c}_{u}, T\right]\right\rangle\right] \\
& \alpha_{n}(u, R, T)=\left[\left\langle\alpha_{n-1}\left(u .0, a_{u},\left[b_{u}, R\right]\right) ; c_{u}\right\rangle,\left\langle\bar{a}_{u} ; \alpha_{n-1}\left(u .1, \bar{b}_{u},\left[\bar{c}_{u}, T\right]\right)\right\rangle\right], \text { if } n>0,
\end{aligned}
$$

where $u \in \mathrm{N}^{*}, a \neq b \neq c \neq a$ and $R, T$ are flat par structures. A structure of the form $\alpha_{n}(u, R, T)$ is called an $\alpha_{n}$-structure. We denote with $S_{n}$ the structure $\alpha_{n}(0, \circ, \circ)$.

Note that the use of indices in naming atoms in the above definition is just to guarantee the pairwise-distinctness of atoms in the generated structures. In the following discussions, we shall often drop the indices when it is understood from context that the atoms under consideration are pairwise distinct.

Recall from the proof in Section 4 that proof search for the structure $S_{0}$ (and, as we shall see later, any other $S_{n}$ ) must start with certain innermost redexes in the structure. A way to show the necessity of deep inference is to show that for each $n$, any rules that modify the structural relations in $S_{n}$ but leave the innermost redexes unchanged would result in an unprovable structure. However, this would leave us with infinite possible inference rules to consider. We therefore need a finite characterization of inference rules, which is complete for the class of structures under consideration. We use the relation web representation for this purpose. Recall that a (shallow) inference rule, applied to a structure, modifies structural relations between atom occurrences in the structure. For instance, the following rule

$$
\rho \frac{([R, T], U)}{[R,(T, U)]}
$$

can be seen, at the relation web level, as the instruction (reading the rule bottom up): change all the structural relations between the atom occurrences in $R$ and $U$ to $\uparrow$. Of course, one cannot arbitrarily modify the structural relations in a relation web, since the resulting web may not correspond to a valid structure. The characterization of structures in Theorem 5.2 is used to rule out certain invalid operations on the relation webs (i.e., by showing that certain forbidden configurations would result from them). However, this characterization alone is not enough for showing the necessity of deep inference, since they deal with structures in general, while we require as well characterization of provable structures. The following lemma states that there are additional configurations that are absent from provable structures. 
Lemma 7.3. Let $S$ be a structure consisting of pairwise distinct atoms. If $S$ is provable in BV then the relation web of $S$ does not contain any of the following configurations:

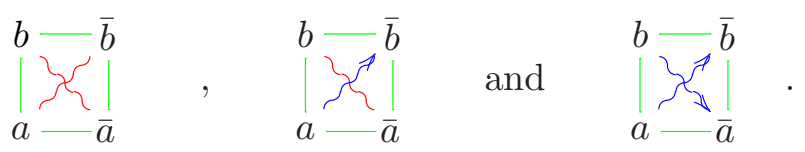

Proof. Suppose that $S$ is provable in BV but $\mathbf{w}_{S}$ contains such configurations, i.e., there exists atoms $a, \bar{a}, b, \bar{b} \in$ occ $S$ that are interrelated as in one of the configurations above. Take any proof $\Pi \pi_{S}$ BV . By repeated applications of Proposition 3.5 we can remove all the atom occurrences from $\Pi$, except for the atoms $\{a, b, \bar{a}, \bar{b}\}$, and obtain the proof $\frac{\Pi^{\prime}}{\|_{S^{\prime}}}$ BV . The relation web of $S^{\prime}$ must be in one of the configurations above, which correspond to the structures $[(a, \bar{b}),(\bar{a}, b)],[\langle a ; \bar{b}\rangle,(\bar{a}, b)]$ and $[\langle a ; \bar{b}\rangle,\langle b ; \bar{a}\rangle]$, respectively. As can be easily checked, none of these structures are provable in $\mathbf{B V}$, which contradicts the fact that $S^{\prime}$ is provable.

We illustrate here how the characterizations of (provable) structures can be used to show that $S_{0}$ can be proved only by modifying the innermost redexes first. This will be shown using in particular the square and triangular properties and the results from Lemma [7.3. Lemma [6.6] and Definition 6.1] The relation web of $S_{0}$ (the indexes are omitted) is the following:

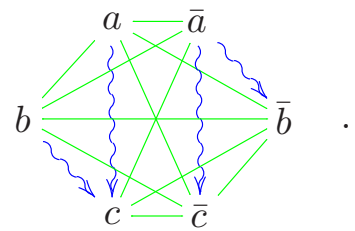

Suppose, for example, there is a proof of $S_{0}$ that starts with a rule $\rho$ of depth 1 . Then by Lemma [6.6] the structural relations $a \downarrow b$ and $\bar{b} \downarrow \bar{c}$ must be preserved by $\rho$ since they correspond to substructures at depth 2. Assuming the application of $\rho$ is non-trivial, some other structural relations must be changed. The changing, however, must preserve provability, which means the par relations between dual atoms must also be preserved. Given these constraints, any other changes to the structural relations will either result in a violation of a certain structural property or destroy the provability. For example, if we change $a \downarrow \bar{c}$ to $a \uparrow \bar{c}$, the triangular and square properties will be violated:

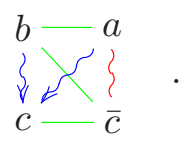

This idea can be generalised to the case

$$
S_{n}=\left[\langle[P\{a\}, Q\{b\}] ; c\rangle,\left\langle\bar{a} ;\left[P^{\prime}\{\bar{b}\}, Q^{\prime}\{\bar{c}\}\right]\right\rangle\right] .
$$

If we preserve all the structural relations inside $[P\{a\}, Q\{b\}]$ and $\left[P^{\prime}\{\bar{b}\}, Q^{\prime}\{\bar{c}\}\right]$, then all other structural relations cannot be changed without changing the provability of $S_{n}$. This idea is formalized in Lemma 7.8 and Lemma 7.9 . 
Lemma 7.4. For every flat par structures $R$ and $T$, and for every $u \in \mathrm{N}^{*}$ there exists a derivation from $[R, T]$ to $\alpha_{n}(u, R, T)$ in $\mathbf{B V}$.

Proof. Base Case: $\alpha_{0}(u, R, T)=\left[\left\langle\left[a_{u}, b_{u}, R\right] ; c_{u}\right\rangle,\left\langle\bar{a}_{u} ;\left[\bar{b}_{u}, \bar{c}_{u}, T\right]\right\rangle\right]$. The derivation needed for this case is given in Figure 5 (modulo some renaming of atoms).

$[U, V]$

Inductive Case: Assume that $\begin{gathered}\| \mathbf{B V} \\ \alpha_{i}(v, U, V)\end{gathered}$ for all $i<n$, for all $v \in \mathrm{N}^{*}$ and for all flat par structures $U, V$. Then

$$
\begin{gathered}
{[R, T]} \\
\Delta_{0} \| \\
{\left[\left\langle\left[a_{u}, b_{u}, R\right] ; c_{u}\right\rangle,\left\langle\bar{a}_{u} ;\left[\bar{b}_{u}, \bar{c}_{u}, T\right]\right\rangle\right]} \\
\Delta_{1} \| \\
{\left[\left\langle\left[a_{u}, b_{u}, R\right] ; c_{u}\right\rangle,\left\langle\bar{a}_{u} ; \alpha_{n-1}\left(u .1, \bar{b}_{u},\left[\bar{c}_{u}, T\right]\right)\right\rangle\right]} \\
\Delta_{2} \| \\
\alpha_{n}(u, R, T)=\left[\left\langle\alpha_{n-1}\left(u .0, a_{u},\left[b_{u}, R\right]\right) ; c_{u}\right\rangle,\left\langle\bar{a}_{u} ; \alpha_{n-1}\left(u .1, \bar{b}_{u},\left[\bar{c}_{u}, T\right]\right)\right\rangle\right]
\end{gathered}
$$

where $\Delta_{0}$ is the derivation constructed in the base case, and $\Delta_{1}$ and $\Delta_{2}$ are derivations obtained from induction hypothesis.

Lemma 7.5. For every $n, S_{n}$ is provable in $\mathbf{B V}$.

Proof. Follows immediately from Lemma 7.4 since $S_{n}=\alpha_{n}(0, \circ, \circ)$.

Lemma 7.6. There are no substructures of the form $[R, \bar{R}]$ in $S_{n}$.

Proof. Base Case: $S_{0}=\alpha_{0}(0, \circ, \circ)=\left[\left\langle\left[a_{0}, b_{0}\right] ; c_{0}\right\rangle,\left\langle\bar{a}_{0} ;\left[\bar{b}_{0}, \bar{c}_{0}\right]\right\rangle\right]$, obvious.

Inductive Case: Assume that $S_{n-1}=\alpha_{n-1}(0, \circ, \circ)$ has no substructures of the form $[R, \bar{R}]$. The change of index from $\alpha_{n-1}(0, \circ, \circ)$ to $\alpha_{n-1}(u, \circ, \circ)$ does not affect the form of the structure, since all the indexed atoms are distinct by definition. The addition of new atoms to $\alpha_{n-1}(u, \circ, \circ)$, i.e., $\alpha_{n-1}(u, U, V)$, does not introduce any new dual substructures, provided that $U$ and $V$ have no dual atoms and consist of different atoms than $\alpha_{n-1}(u, \circ, \circ)$. Therefore the structure

$$
S_{n}=\alpha_{n}(0, \circ, \circ)=\left[\left\langle\alpha_{n-1}\left(0.0, a_{0}, b_{0}\right) ; c_{0}\right\rangle,\left\langle\bar{a}_{0} ; \alpha_{n-1}\left(0.1, \bar{b}_{0}, \bar{c}_{0}\right)\right\rangle\right]
$$

does not contain any substructures of the form $[R, \bar{R}]$ either.

Lemma 7.7. Every occurrence of $\alpha_{0}$-structure in $\alpha_{n}(u, R, T)$ is at depth $2 n$.

Proof. The base case is obvious. For the inductive case, assume that every occurrence of $\alpha_{0}$-structure in $\alpha_{n-1}(w, P, Q)$ is at depth $2(n-1)$. Since

$$
\alpha_{n}(u, R, T)=\left[\left\langle\alpha_{n-1}\left(u .0, a_{u},\left[b_{u}, R\right]\right) ; c_{u}\right\rangle,\left\langle\bar{a}_{u} ; \alpha_{n-1}\left(u .1, \bar{b}_{u},\left[\bar{c}_{u}, T\right]\right)\right\rangle\right],
$$

the depth of each $\alpha_{0}$-structure in $\alpha_{n}(u, R, T)$ is $2(n-1)+2=2 n$.

The next three lemmas are crucial to the proof by contradiction in the main theorem on the necessity of deep inference.

Lemma 7.8. Let $\rho \frac{T}{R} \quad$ be a proof in a shallow system $\mathscr{S}$ where $\rho$ is a shallow rule. If $\mathscr{S}$ is equivalent to $\mathbf{B V}$ and the following conditions hold: 
(a): $R$ consists of pairwise distinct atoms,

(b): $\mathbf{w}_{R}$ contains the following configuration:

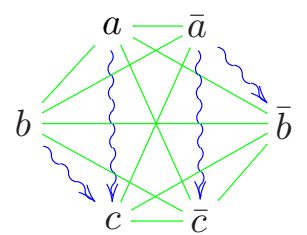

for some pairwise distinct atoms $a, b$ and $c$,

(c): $a \downarrow_{T} b$ and $\bar{b} \downarrow_{T} \bar{c}$,

then the configuration (i) is preserved by $\rho$.

Proof. Since $T$ is provable, the par links between dual atoms in $R$ must be preserved by $\rho$. This, together with conditions (a)-(c), give us the following configuration in $\mathbf{w}_{T}$ :

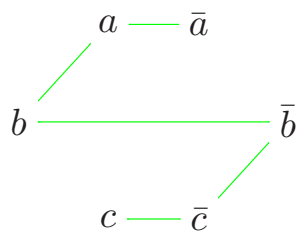

We assume above that $\mathscr{S}$ is equivalent to BV. By Definition 6.4 and Definition 6.3, we have that $R \prec T$. This means, by Definition 6.1 that if $x \triangleleft_{R} y$ or $x \uparrow_{R} y$ then it cannot be the case that $x \downarrow_{T} y$. Therefore it must hold in $\mathbf{w}_{T}$ :

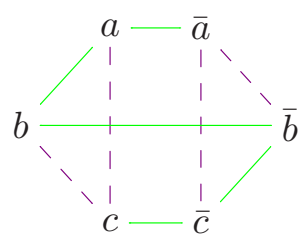

(Recall that the dotted line indicates the negation of the structural relation it represents, in this case, the par link.) We first show that all par relations $x \downarrow_{R} y$ in (i) must be preserved, and then, based on this result we prove that any seq relations $x \triangleleft_{R} y$ in (i) must also be preserved.

1.: $a \downarrow_{T} \bar{a}, b \downarrow_{T} \bar{b}, c \downarrow_{T} \bar{c}, a \downarrow_{T} b$ and $\bar{b} \downarrow_{T} \bar{c}$, see diagram (ii).

2.: $a \downarrow_{T} \bar{b}$. Suppose that $\neg\left(a \downarrow_{T} \bar{b}\right)$. Adding this information to (ii) we have in $\mathbf{w}_{T}$ :

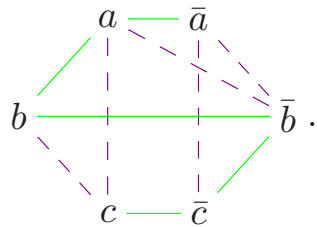

Take the atoms $a, \bar{a}, \bar{b}$ and $\bar{c}$. By the inverse square property (Corollary [5.3), we infer that $\neg\left(a \downarrow_{T} \bar{c}\right)$, as shown in the following figure:

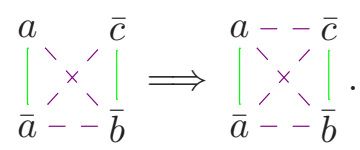


Now we combine this information with (ii.1):

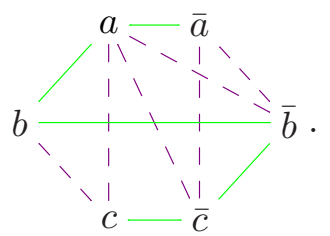

Take the atoms $b, \bar{b}, a$ and $c$. By the square property (Theorem [5.2), $b \downarrow_{T} \bar{c}$ as shown below:

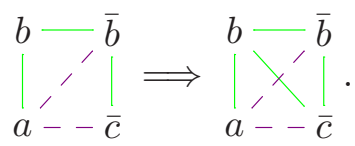

This together with (ii.2) yield:

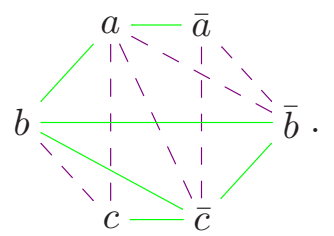

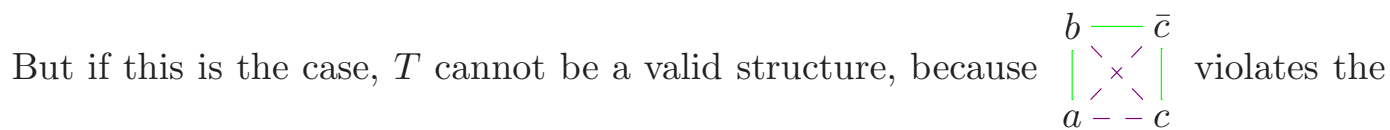
square property. Therefore $a \downarrow \bar{b}$ must hold in $T$.

3.: $a \downarrow \bar{c}$ in $T$. Suppose that $\neg(a \downarrow \bar{c})$, following a similar reasoning as in Case 2, we have (s.p. stands for the (inverse) square property):

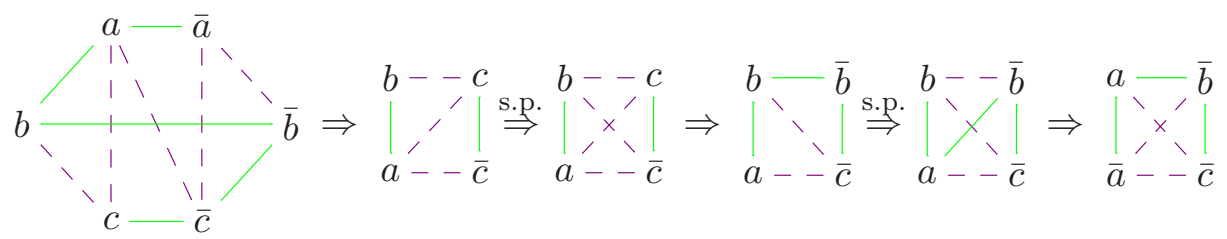

4.: $b \downarrow_{T} \bar{a}$. Suppose $\neg\left(b \downarrow_{T} \bar{a}\right)$. It follows from the results established in cases 1-3 that in $\mathbf{w}_{T}$ :

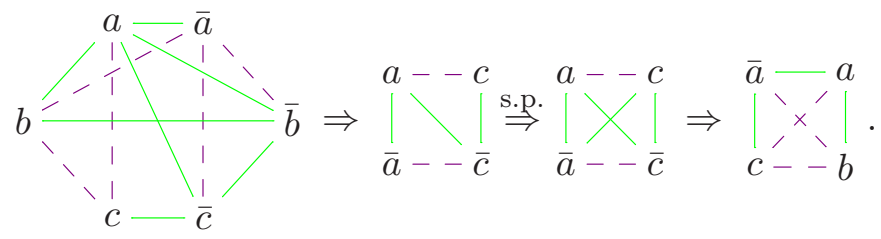

5.: $b \downarrow_{T} \bar{c}$. This case is analogous to case 2. That is if we suppose that $\neg\left(b \downarrow_{T} \bar{c}\right)$, then the relation web:

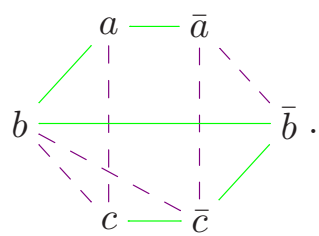

is isomorphic to (ii.1) in case 2 . 
6.: $\bar{a} \downarrow_{T} c$. Suppose $\neg\left(\bar{a} \downarrow_{T} c\right)$. Since $a \downarrow_{T} c$, as shown in Case 3 , we have the following forbidden configuration:

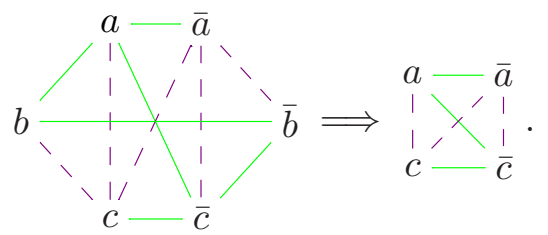

We are left with the relations $a \triangleleft_{R} c, b \triangleleft_{R} c, \bar{a} \triangleleft_{R} \bar{b}$ and $\bar{a} \triangleleft_{R} \bar{c}$. Since the structural relation $\triangleleft$ can change only to $\uparrow$, there are 16 possible configurations to consider. By applying the triangular property, it is easy to see that $a \uparrow_{T} c$ iff $b \uparrow_{T} c$, and that $\bar{a} \uparrow_{T} \bar{b}$ iff $\bar{a} \uparrow_{T} \bar{c}$. This is the case because otherwise we would have the following forbidden configurations:

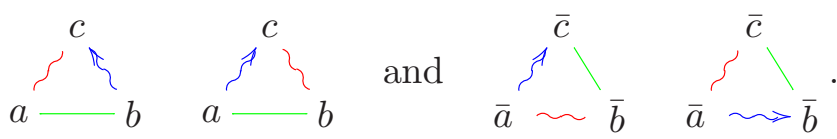

Therefore, we need only to consider the following three cases.

7.: $a \uparrow_{T} c, b \uparrow_{T} c, \bar{a} \triangleleft_{T} \bar{b}$ and $\bar{a} \triangleleft_{T} \bar{c}$.

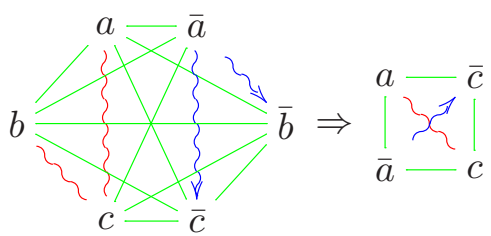

8.: $a \triangleleft_{T} c, b \triangleleft_{T} c, \bar{a} \uparrow_{T} \bar{b}$ and $\bar{a} \uparrow_{T} \bar{c}$.

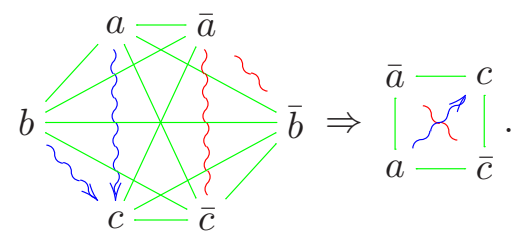

9.: $a \uparrow_{T} c, b \uparrow_{T} c, \bar{a} \uparrow_{T} \bar{b}$ and $\bar{a} \uparrow_{T} \bar{c}$.

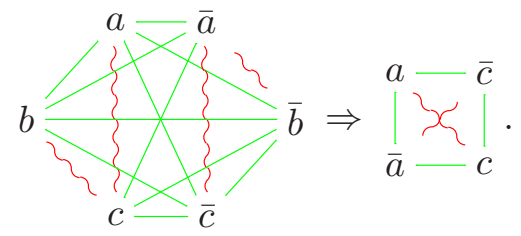

The configurations in cases 7, 8 and 9 are forbidden by Lemma 7.3 , which means that the relations $a \triangleleft_{R} c, b \triangleleft_{R} c, \bar{a} \triangleleft_{R} \bar{b}$ and $\bar{a} \triangleleft_{R} \bar{c}$ must be preserved by $\rho$. Therefore, the configuration (i) must hold in $T$. 
Lemma 7.9. Let $\rho \frac{U}{S\{P\}}$ be a proof in a shallow system $\mathscr{S}$ where $\rho$ is a shallow rule, $P$ is an $\alpha_{n}$-structure and $S\{P\}$ consists of pairwise distinct atoms. If $\mathscr{S}$ is equivalent to $\mathbf{B V}$ and all the structural relations in $\alpha_{n-1}$-substructures of $P$ are preserved by $\rho$ then all the structural relations in $P$ are also preserved by $\rho$.

Proof. Let us recall the definition of $\alpha_{n}$-structure:

$$
P=\alpha_{n}(u, R, T)=\left[\left\langle\alpha_{n-1}\left(u .0, a_{u},\left[b_{u}, R\right]\right) ; c_{u}\right\rangle,\left\langle\bar{a}_{u} ; \alpha_{n-1}\left(u .1, \bar{b}_{u},\left[\bar{c}_{u}, T\right]\right)\right\rangle\right] .
$$

All the structural relations in $\alpha_{n-1}\left(u .0, a_{u},\left[b_{u}, R\right]\right)$ (likewise, $\left.\alpha_{n-1}\left(u .1, \bar{b}_{u},\left[\bar{c}_{u}, T\right]\right)\right)$ are preserved by $\rho$.

Let the variables $x, y$ stand for atoms in occ $P$. The following case analyses show that the structural relation between $x$ and $y$, for all possible values of $x$ and $y$, must be preserved by $\rho$ as a consequence of the structural properties of the underlying relation webs of $S\{P\}$ and $U$. We shall use the following abbreviation

$$
P_{1}=\alpha_{n-1}\left(u .0, a_{u},\left[b_{u}, R\right]\right) \text {, and } P_{2}=\alpha_{n-1}\left(u .1, \bar{b}_{u},\left[\bar{c}_{u}, T\right]\right),
$$

in the case analyses below.

1.: $x, y \in \operatorname{occ} P_{1}$, or $x, y \in \operatorname{occ} P_{2}$, follows immediately from the condition of this lemma.

2.: $x \in \operatorname{occ} P_{1}, y=c_{u}$. It holds in $P: x \triangleleft y$.

a.: $x \in\left\{a_{u}, b_{u}\right\}$. The structural relations between the atoms $a_{u}, b_{u}, c_{u}, \bar{a}_{u}, \bar{b}_{u}$ and $\bar{c}_{u}$ in $P$ are

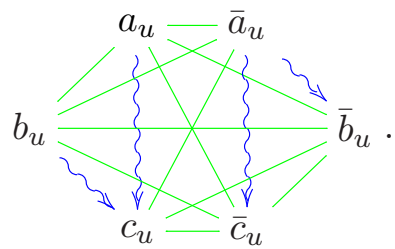

Note that since $a_{u}, b_{u} \in \operatorname{occ} P_{1}$ and $\bar{b}_{u}, \bar{c}_{u} \in \operatorname{occ} P_{2}$, and since by assumption all structral relations inside $P_{1}$ and $P_{2}$ are unchanged by $\rho$, we have $a_{u} \downarrow_{U} b_{u}$ and $\bar{b}_{u} \downarrow_{U} \bar{c}_{u}$. Therefore, by Lemma 7.8, the above configuration is preserved by $\rho$.

b.: $x \notin\left\{a_{u}, b_{u}\right\}$. Then $x \downarrow_{P} a_{u}$ or $x \downarrow_{P} b_{u}$, because by Definition 7.2

$$
\alpha_{n-1}\left(u .0, a_{u},\left[b_{u}, R\right]\right)=\left[Q_{1}\left\{a_{u}\right\}, Q_{2}\left\{b_{u}\right\}\right],
$$

for some structure contexts $Q_{1}\{\}$ and $Q_{2}\{\}$. Depending on whether $x \downarrow_{P} b_{u}$ or $x \downarrow_{P} c_{u}$, one of the following configurations must hold in $P$ :

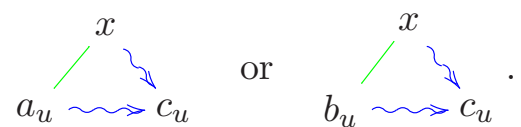

The relations between $x$ and $\left\{a_{u}, b_{u}\right\}$ are preserved by $\rho$ (they all belong to $P_{1}$ ), so it holds in $U: x \downarrow_{U} a_{u}$ or $x \downarrow_{U} b_{u}$. The structural relations $a_{u} \triangleleft_{U} c_{u}$ and $b_{u} \triangleleft_{U} c_{u}$ must hold as shown in Case 2.a. The structural relation $x \triangleleft_{U} c_{u}$ must hold in $U$, because otherwise $x \uparrow_{U} c_{u}$ by Definition 6.1 and one of the following 
forbidden configuration would occur in $U$, depending on whether $x \downarrow_{U} a_{u}$ or $x \downarrow_{U} b_{u}$.

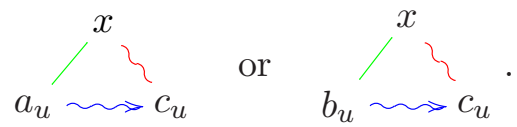

3.: $x \in \operatorname{occ} P_{2}, y=\bar{a}_{u}$. It holds in $P: y \triangleleft_{P} x$.

a.: $x \in\left\{\bar{b}_{u}, \bar{c}_{u}\right\}$, see Case 2.a.

b.: $x \notin\left\{\bar{b}_{u}, \bar{c}_{u}\right\}$, then $x \downarrow_{U} \bar{b}_{u}$ or $x \downarrow_{U} \bar{c}_{u}$. Using a similar argument as in Case 2.b, we establish that the structural relation $\bar{a}_{u} \triangleleft_{U} x$ must hold, because otherwise the following forbidden configurations would occur in $U$ :

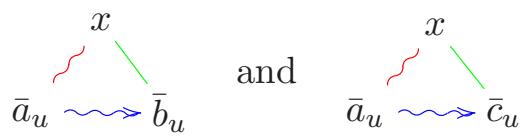

which correspond to the cases $x \downarrow_{U} \bar{b}_{u}$ and $x \downarrow_{U} \bar{c}_{u}$, respectively.

4.: $x \in \operatorname{occ}\left\langle P_{1} ; c_{u}\right\rangle, y \in \operatorname{occ}\left\langle\bar{a}_{u} ; P_{2}\right\rangle$.

a.: $x \in\left\{a_{u}, b_{u}, c_{u}\right\}, y \in\left\{\bar{a}_{u}, \bar{b}_{u}, \bar{c}_{u}\right\}$, see Case 2.a.

b.: $x \in\left\{a_{u}, b_{u}, c_{u}\right\}, y \notin\left\{\bar{a}_{u}, \bar{b}_{u}, \bar{c}_{u}\right\}$. Since $y \in$ occ $P_{2}$, by assumption we have either $y \downarrow_{U} \bar{b}_{u}$ or $y \downarrow_{U} \bar{c}_{u}$. The strutural relations between atoms in $\left\{x, \bar{a}_{u}, \bar{b}_{u}, \bar{c}_{u}\right\}$ are preserved by $\rho$, as shown in Case 2.a, and the structural relations between atoms in $\left\{y, \bar{a}_{u}, \bar{b}_{u}, \bar{c}_{u}\right\}$ are also preserved by $\rho$, as shown in Case 1 and Case 3. Therefore, if we change the relation $x \downarrow_{P} y$ to $\neg\left(x \downarrow_{U} y\right)$, the square property will be violated, as shown in the following diagrams:

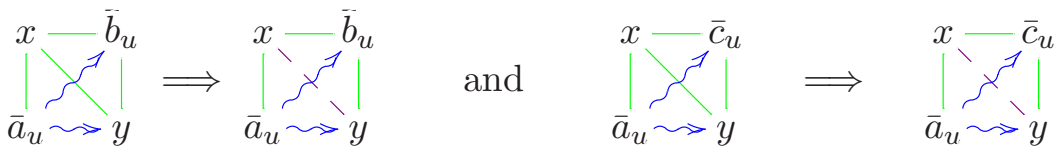

which correspond to the cases $y \downarrow_{U} \bar{b}_{u}$ and $y \downarrow_{U} \bar{c}_{u}$, respectively.

c.: $x \notin\left\{a_{u}, b_{u}, c_{u}\right\}, y \in\left\{\bar{a}_{u}, \bar{b}_{u}, \bar{c}_{u}\right\}$. Then it holds in $U: x \downarrow_{U} a_{u}$ or $x \downarrow_{U} b_{u}$. The structural relations between atoms in $\left\{x, a_{u}, b_{u}, c_{u}\right\}$ must be preserved by $\rho$, as shown in Case 1 and 2, and the structural relations between atoms in $\left\{y, a_{u}, b_{u}, c_{u}\right\}$ must also be preserved as shown in Case 2.a. The changing of $x \downarrow_{P} y$ to $\neg\left(x \downarrow_{U} y\right)$ leads to the violation of square property as shown in the following diagrams:

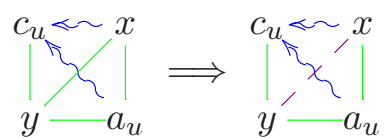

and

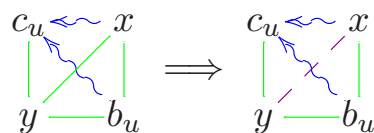

which correspond to the cases $x \downarrow_{U} a_{u}$ and $x \downarrow_{U} b_{u}$, respectively.

d.: $x \notin\left\{a_{u}, b_{u}, c_{u}\right\}, y \notin\left\{\bar{a}_{u}, \bar{b}_{u}, \bar{c}_{u}\right\}$. The structural relations $\bar{a}_{u} \downarrow_{P} c_{u}, x \triangleleft_{P} c_{u}$, $\bar{a}_{u} \triangleleft_{P} y, c_{u} \downarrow_{P} y$ and $\bar{a}_{u} \downarrow_{P} x$ are preserved by $\rho$ as shown in Case 3, 4.a, 4.b and 4.c. Therefore, if the relation $x \downarrow_{P} y$ is changed to $\neg\left(x \downarrow_{U} y\right)$, the square 
property would be violated.

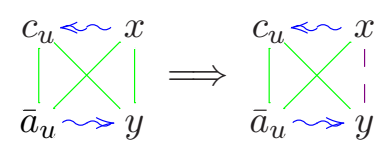

Lemma 7.10. Let $\frac{\prod}{S\{P\}}$ be a proof in a shallow system $\mathscr{S}$, where $P$ is an $\alpha_{n}$-struture and $\rho$ is a shallow rule. If $\mathscr{S}$ is equivalent to $\mathbf{B V}$ and all the structural relations in every $\alpha_{0^{-}}$ substructure of $P$ are preserved by $\rho$, then all the structural relations in $P$ are also preserved by $\rho$.

Proof. The case where $P=\alpha_{0}(u, R, T)$ is obvious. So suppose that

$$
P=\alpha_{n}(u, R, T)=\left[\left\langle\alpha_{n-1}\left(u .0, a_{u},\left[b_{u}, R\right]\right) ; c_{u}\right\rangle,\left\langle\bar{a}_{u} ; \alpha_{n-1}\left(u .1, \bar{b}_{u},\left[\bar{c}_{u}, T\right]\right)\right\rangle\right]
$$

By inductive hypothesis, the structural relations in $\alpha_{n-1}$ substructures of $P$ are preserved by $\rho$. This means, by Lemma 7.9, that all the structural relations in $P$ must also be preserved by $\rho$.

Theorem 7.11. No shallow system can be equivalent to $\mathbf{B V}$.

Proof. Suppose that there exists a shallow system $\mathscr{S}$ which is equivalent to BV. Let $n$ be the depth of $\mathscr{S}$. Consider the structure $S_{n+1}$. By Lemma $7.5 S_{n+1}$ is provable in BV. Since $\mathscr{S}$ is equivalent to BV, there must exist also a proof

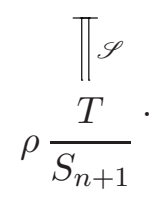

We can assume without loss of generality that the instance of $\rho$ above is non-trivial, i.e., $T \neq S_{n+1}$.

The rule $\rho$ cannot be an interaction rule since by Lemma [7.6 there are no substructures of the form $[R, \bar{R}]$ in $S_{n+1}$. Therefore it must be a shallow rule with depth at most $n$. From Lemma 7.7 all the $\alpha_{0}$-substructures in $S_{n+1}$ are at depth $2(n+1)$, so all the structural relations in any $\alpha_{0}$-substructures of $S_{n+1}$ must be preserved by $\rho$. But by Lemma 7.10 . this implies that all the structural relations in $S_{n+1}$ must also be preserved by $\rho$, that is, $T=S_{n+1}$, contrary to the assumption that the application of $\rho$ is non-trivial. Therefore, $S_{n+1}$ is not provable in $\mathscr{S}$ and consequently, $\mathscr{S}$ cannot be equivalent to BV.

\section{Conclusion}

We have shown that deep inference is necessary for System BV, that is, any restriction on the depth of the applicability of the rules in BV would result in a strictly less expressive logical system. This result extends to all conservative extensions of $\mathbf{B V}$, for instance, the extension of BV with the exponential modalities of linear logic GS01. This result also shows in particular that finding a simple sequent system for $\mathbf{B V}$ is challenging, to say the 
least. At the more conceptual level, we hope that it gives sufficient evident that the calculus of structures, especially deep-inference, is a non-trivial departure from sequent calculus as a formalism for presenting logics.

System BV is not the only logical system that incorporates both commutative and noncommutative connectives. Retoré's Pomset logic Ret99 achieves the same thing. However, Pomset logic is based on proof-nets, and its sequentialisation is still an open question. Retoré uses a presentation of proof-nets called the $R \mathscr{E} B$-directed cographs, which are essentially relation webs where the par links are implicit except when they connect dual atoms. The structure $S_{0}$ defined in Section 7 , for example, corresponds to the directed cographs:

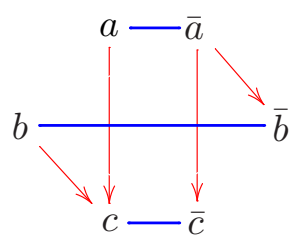

where the par links are omitted except those connecting dual atoms. The connection between BV and Pomset logic has been extensively discussed in Str03, where it is shown a sound translation from BV to Pomset logic, i.e., every provable structure (hence, relation webs) in BV corresponds to a proof net in Pomset logic. Our result on the necessity of deep inference for BV therefore carries over to Pomset logic. That is, certain types of sequent systems (i.e., those which can be presented as shallow systems) can be ruled out in the search for the sequentialization of Pomset logic.

\section{ACKNOWLEDGMENTS}

I would like to thank Alessio Guglielmi, Kai Bruennler, Paola Bruscoli, Lutz Strassburger and the anonymous referees for their useful comments and suggestions on earlier drafts of this paper. An earlier version of this paper has appeared as a technical report Tiu01] at TU Dresden.

\section{REFERENCES}

[Bel82] N. D. Belnap. Display logic. Journal of Philosophical Logic, 11:375-417, 1982.

[Brü03] Kai Brünnler. Deep Inference and Symmetry in Classical Proofs. PhD thesis, TU Dresden, September 2003.

[BT01] Kai Brünnler and Alwen Fernanto Tiu. A local system for classical logic. In R. Nieuwenhuis and A. Voronkov, editors, LPAR 2001, volume 2250 of Lecture Notes in Artificial Intelligence, pages 347-361. Springer-Verlag, 2001.

[Gen69] Gerhard Gentzen. Investigations into logical deduction. In M. E. Szabo, editor, The Collected Papers of Gerhard Gentzen, pages 68-131. North-Holland, Amsterdam, 1969.

[Gir87] Jean-Yves Girard. Linear logic. Theoretical Computer Science, 50:1-102, 1987.

[GS01] Alessio Guglielmi and Lutz Straßburger. Non-commutativity and MELL in the calculus of structures. In L. Fribourg, editor, CSL 2001, volume 2142 of Lecture Notes in Computer Science, pages 54-68. Springer-Verlag, 2001.

[Gug99] Alessio Guglielmi. A calculus of order and interaction. Technical Report WV-99-04, Dresden University of Technology, 1999.

[Gug02] Alessio Guglielmi. A system of interaction and structure. Technical Report WV-02-10, Technische Universität Dresden, 2002. To appear in ACM Transactions on Computational Logic.

[Möh89] Rolf H. Möhring. Computationally tractable classes of ordered sets. In I. Rival, editor, Algorithms and Order, pages 105-194. Kluwer Academic Publishers, Dordrecht, 1989. 
[OP99] P. O'Hearn and D. Pym. The logic of bunched implications. Bulletin of Symbolic Logic, 5(2):215243, June 1999.

[Ret97] Christian Retoré. Pomset logic: A non-commutative extension of classical linear logic. In $\mathrm{Ph}$. de Groote and J. R. Hindley, editors, TLCA'97, volume 1210 of Lecture Notes in Computer Science, pages 300-318, 1997.

[Ret99] Christian Retoré. Pomset logic as a calculus of directed cographs. In V. M. Abrusci and C. Casadio, editors, Dynamic Perspectives in Logic and Linguistics, pages 221-247. Bulzoni, Roma, 1999. Also available as INRIA Rapport de Recherche RR-3714.

[Str03] Lutz Straßburger. Linear Logic and Noncommutativity in the Calculus of Structures. PhD thesis, Technische Universität Dresden, 2003.

[Tiu01] Alwen Tiu. Properties of a logical system in the calculus of structures. Technical Report WV-01-06, Dresden University of Technology, 2001.

[TS96] A.S. Troelstra and H. Schwichtenberg. Basic Proof Theory. Cambridge University Press, 1996. 